\title{
Minimally Invasive en bloc Excision of Rare Hemorrhagic Discal Cysts Using Unilateral Biportal Endoscopic Technique: A Report of Two Cases
}

\author{
Chun-Hung Lin ${ }^{1}$, Jwo-Luen Pao ${ }^{1,2}$ \\ ${ }^{1}$ Department of Orthopedic Surgery, Far Eastern Memorial Hospital, New Taipei City, Taiwan \\ ${ }^{2}$ General Education Center, Longhwa University of Science and Technology, Taoyuan, Taiwan
}

Received: October 12, 2021

Accepted: November 29, 2021

Corresponding Author:

Jwo-Luen Pao, MD

Department of Orthopedic Surgery,

Far Eastern Memorial Hospital, No.

21, Sec. 2, Nanya South Road,

Banqiao District, New Taipei 220,

Taiwan

Tel: +886-2-89667000 (ext. 2888)

Fax: +886-2-77282894

E-mail: jwoluenpao@gmail.com
Hemorrhagic discal cyst (HDC) is a rare cause for low back pain and radiculopathy. Although there is no treatment guideline in the literature, it is believed that the surgi-cal excision showed better treatment results than the conservative treatment. In this report, we described two patients with HDC who were treated using the minimally invasive unilateral biportal endoscopic (UBE) technique. Two patients with the rare HDC in their lumbosacral spines came to our clinic due to low back pain and severe sciatica after failure of conservative treatment. Both patients' MRI revealed a cystic mass mimic a ruptured lumbar disc herniation with compression of the nerve root. The cysts were excised using UBE technique. The features of UBE technique are two small surgical wounds, minimal muscle dissection, continuous normal saline irrigation, excellent hemostasis, and a crystal clear and magnified endoscopic surgical field. These features enable meticulous dissection, en bloc excision of the cyst, and reduce the risks of dural tears or nerve root injuries. Both patients had very good symptoms relief and quick recovery after the surgery with no complications. The UBE technique is a safe, effective, and excellent surgical treatment option for patients with HDC in the lumbosacral spine.

Key Words: Hemorrhagic discal cyst, Unilateral biportal endoscopic technique, Endoscopic spine surgery, Minimally invasive surgery

\section{INTRODUCTION}

The hemorrhagic discal cyst (HDC) is a rare intraspinal extradural cystic lesion that connects with the lumbar intervertebral discs [1]. The pathophysiology of the HDC is unclear. Most of the reported cases are relatively young and active males possibly associated with trauma histories [2].

The clinical presentations of HDC are very much like the lumbar disc herniation. The differential diagnosis between them was not difficult under the magnetic resonance images
(MRI). In the reported cases, the surgical excision of the cyst seemed to be a better option than the conservative treatment [3]. While the traditional open surgery was effective, the major drawbacks were massive soft tissue dissection and excessive destruction of the bony structures. Furthermore, the incidental dural tears and nerve root injuries with traditional open surgery were also reported due to severe adhesion between the cysts and the neural structures $[4,5]$.

Unilateral biportal endoscopic surgery (UBE) has been performed for more than 10 years [6]. This minimally invasive

Copyright @ 2022 Korean Minimally Invasive Spine Surgery Society

This is an Open Access article distributed under the terms of the Creative Commons Attribution Non-Commercial License (http://creativecommons.org/licenses/by-nc/4.0/) which permits unrestricted non-commercial use, distribution, and reproduction in any medium, provided the original work is properly cited. 
endoscopic technique has been successfully applied in various treatments with conditions including lumbar disc herniation and degenerative lumbar spinal stenosis [7,8]. However, UBE has not been used in the treatment of lumbar HDC in the past. In this case report, we shared two successful treatment cases of HDC using the UBE technique.

\section{CASE REPORT}

\section{Case 1}

The first case was a 41-year-old man with no systematic disease. He was referred to our hospital due to his low back pain, intolerable sciatica in his right leg, and difficulty in walking after lifting a heavy object 2 months ago. He had severe radicular leg pain originated from his right buttock, along the posterior thigh down to posterior calf and foot sole with severe muscle cramps. He also had mild numbness distributed along the S1 dermatome in his right leg. Conservative treatments including NSAIDs and physical therapy did not improve his symptoms at all. The straight leg raising test was positive at 20 degrees in the right leg, and the contralateral straight leg raising test was also positive. Plain radiographs of his lumbar spine showed mild degeneration with no significant disc space narrowing and no segmental instability. The MRI revealed an epidural cystic mass located posteriorly to the L5-S disc space, mimicking a ruptured disc. However, the L5-S disc was essentially normal with only mild degeneration. The right S1 nerve root was displaced posteriorly in the lateral recess. The cyst had low signal intensity on T1-weighted images and high signal intensity on T2-weighted images (Figure 1A).

We planned to remove the cystic mass via right side posterior interlaminar approach using unilateral biportal endoscopic (UBE) technique. Under general anesthesia, the patient was placed prone on the radiolucent Relton-Hall frame. The skin and the surgical field were prepared in the usual manners. The surgical procedure was essentially the same as the UBE discectomy. The spinal level of concerns was localized using the biplanar fluoroscope and marked on the skin. There were two skin incisions through the deep fascia: one smaller about 5-6 $\mathrm{mm}$ for insertion of a 30-degree arthroscope and continuous normal saline irrigation; one larger about $8-10 \mathrm{~mm}$ for the outflow of normal saline and served as the instrumental portal .

The target of the initial laminotomy was the junction of the spinous process and the lower laminar margin of superior vertebra. The two skin incisions located along the medial pedicle line, separated by $2-3 \mathrm{~cm}$. We used serial dilators up to $10 \mathrm{~mm}$ to split the fascia and the paraspinal muscles, enlarge the instrument portal, and gently detach the soft tissues off the interlaminar space. After the inflow of normal saline, a small space was created as the initial surgical field. The whole surgical procedure was performed in a crystal clear and magnified surgical field. The hemostasis was controlled by balancing hydrostatic pressure and using radiofrequency wands to coagulate the bleeders (ArthroCare, Austin, TX, USA); hence, the whole surgical procedure was performed in a crystal clear and magnified surgical field.

This laminotomy was performed using an electric highspeed diamond bur of $4 \mathrm{~mm}$ in diameter (Primado 2; NSK, Fukushima, Japan). Following the laminotomy and the removal of the ligamentum flavum, the dura and the traversing nerve root were identified. The cystic mass was found beneath the shoulder area of the S1 traversing nerve root with severe adhesion between the cyst and the dura. The cyst was well encapsulated, brownish blue in color with severe adhesion to the dura and the nerve root (Figure 1B). There was a fibrotic stalk connecting to the posterior annulus fibrosis of the L5-S disc. The cyst was meticulously dissected and isolated using the blunt dural dissectors and nerve hooks. Then the cyst was removed en bloc by dividing the connecting stalk. A tiny annular defect was identified and closed by thermal annuloplasty using the radiofrequency wand. Histopathological examination of the cyst showed fibrosis and hemosiderin deposition within the cyst wall with no disc materials inside the cyst. Within hours after the operation, the patient noticed great improvements in his symptoms. He was discharged from the hospital on the next day. Four weeks later, free from any pain, the patient had returned to work and resumed his daily activities.

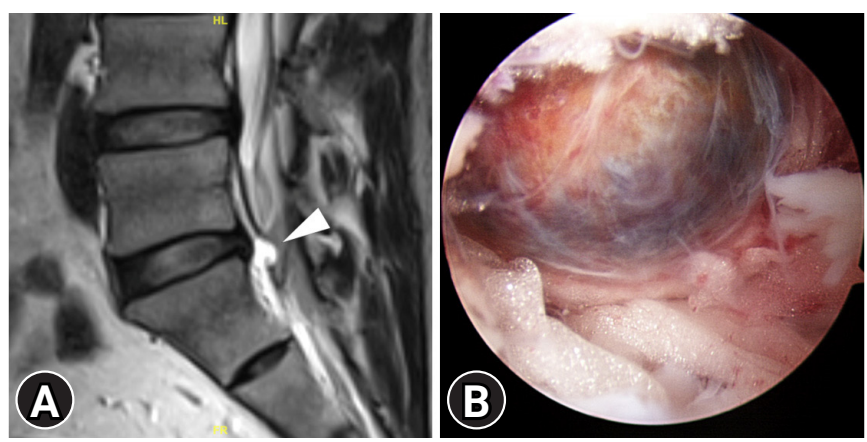

Figure 1. (A) MRI of the lumbar spine showed a cystic mass (white arrow head) mimicking a ruptured disc herniation at L5-S disc level with posterior displacement of the S1 traversing nerve root. (B) Intra-operative photo showed a well-capsulated brownish blue cyst beneath the traversing nerve root. 


\section{Case 2}

The second case was a 25-year-old man who had been suffering from low back pain, radicular pain in his left leg, walking difficulty for 1 year with progressive worsening in the recent 4 months. He had no neurological deficits. The MRI obtained from another hospital revealed an epidural cystic lesion, measured $17 \mathrm{~mm}$ by $11 \mathrm{~mm}$, located at the left side and posterior to the L5 vertebral body at the L5-S disc level. His cystic lesion had high signal intensity on T2-weighted images and low signal intensity on $\mathrm{Tl}$ weighted images (Figure 2A). He had been treated conservatively with NSAIDs, physical therapy, and epidural steroid injections with no improvement at all. Therefore, he was referred to us for further treatment with UBE surgery. The surgical procedures were the same as described for the first case, via left side posterior interlaminar approach. The plane between the cystic lesion and dura was very difficult to get identified because of severe adhesion (Figure 2B, C). However, the surgical field in UBE surgeries was so clear and magnified that we could do delicate dissection without any injury to the dura and the nerve root. The cyst was elastic firm, in dark blue color with no pulsation. Unlike the first case, there was no connecting stalk with the underlying disc. The cyst had a broad base connection with the posterior annulus fibrosis of the L5-S disc but no annular defect could be identified after en bloc excision of the cyst. Histopathological examination reported as a mass with fibrotic cyst wall composed of hemorrhagic deposit with no disc materials inside.

The patient's low back pain and lower leg pain improved immediately after the surgery. He walked very well and was dis- charged from the hospital on the next day. One week after the surgery, the patient returned to his work as a heavy labor with only mild soreness in his left buttock during the initial weeks. Follow-up MRI study after months showed the cyst was excised with a small cyst remnant but no neural compression.

\section{DISCUSSION}

Hemorrhagic discal cyst (HDC) in the lumbar spine is extremely rare that its importance is often overlooked. The clinical presentation and symptoms of HDC are similar to the lumbar disc herniation because of its mass effect and compression to the neural structures. There are three hypotheses regarding its pathogenesis: I. reaction to spinal epidural hematoma; II. a pseudomembrane formation that follows the focal annular tear and disc degeneration; and III. an inflammatory reaction to the herniated disc fragment $[1,9]$. The hemorrhagic content suggested a trauma related mechanism. Computed Tomography (CT) discography and MRI are the most accurate image modalities to detect the HDC. CT discography is able to demonstrate the connecting channel between the cyst and the corresponding intervertebral disc, but it is an invasive procedure. The lesion is not difficult to get detected in the MRI because it shows low signal intensity on T1-weighted images and high signal intensity on T2-weighted images. However, the signal intensity may vary with evolution of the hemorrhage inside the cyst [10].

There was no guideline suggesting the optimal treatment for HDC in the past. Most of the evidences were in the form of case reports or small series $[3,11,12]$. Several studies with conservative treatment showed low satisfaction rate with a low rate of
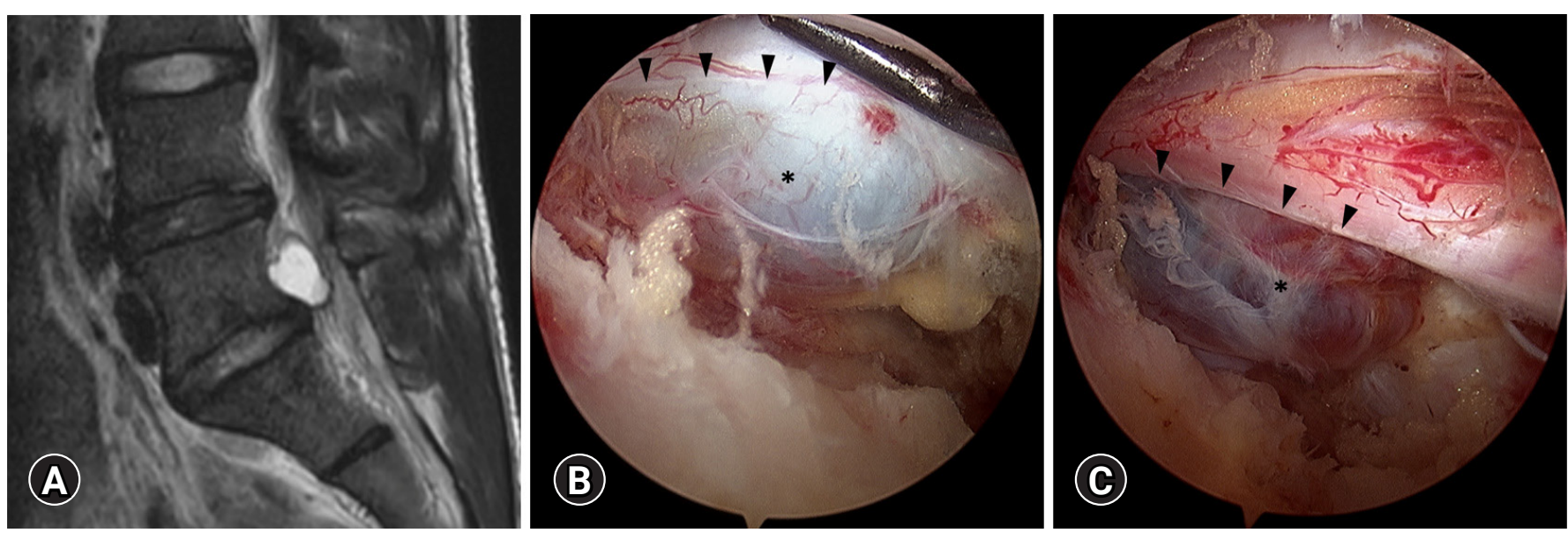

Figure 2. (A) Pre-operative MRI: the broad base cystic mass with posterior displacement of the S1 traversing nerve root. (B) The intra-operative photo: severe adhesion (black arrowheads) with a vague plane between the cyst (asterisk) and the dura. (C) The dark blue hemorrhagic cyst (asterisk) was isolated after meticulous dissection to release the adhesion from the dura and the S1 nerve root. 
spontaneous regression of the cysts. Many conservatively treated cases were ultimately shifted to surgical treatment $[13,14]$. A variety of invasive procedures for the HDC have been suggested, including CT-guided aspiration, laser ablation, and microscopic excision $[15,16]$. The CT-guided aspiration could achieve good clinical results but there still were concerns for recurrence of the cyst. Moreover, it required accurate techniques to avoid injuries to the neural structures [17]. Therefore, the surgical excision of the HDC seemed to be the most reliable way to relieve pain and decompress the neural structures.

Traditional surgical excision of the HDC is generally effective for symptom relief. However, it usually involves a big surgical wound, extensive soft tissue dissection, and excessive bony destruction. All of these issues could lead to atrophy of the paraspinal muscles, chronic post-operative low back pain, and segmental instability. In order to avoid the untoward side effects of the traditional surgery, various minimally invasive surgical techniques were developed. These techniques included the microendoscopic techniques using the tubular retractor system and the percutaneous endoscopic techniques using the uniportal endoscopic system [18]. These minimally invasive techniques shared common advantages including minimal blood loss, minimal postoperative pain, shorter hospitalization, rapid recovery, comparable treatment results with traditional open techniques, preservation of the soft tissues and the paraspinal muscles, and less destruction of the posterior stabilizing structures.

In this report, we used the UBE techniques to en bloc excise the HDC. The follow-up MRI in our cases demonstrated that there was almost no paraspinal muscles damage and very good preservation of the facet joint. Clinical presentations of both patients also showed excellent symptoms relief, quick functional recovery, and no low back pain.

Different from the uniportal endoscopic technique, the UBE technique uses two independent surgical portals: one for the endoscope and the other for the instruments. Handling of the surgical instruments in the UBE technique is not restricted in the endoscopic channel. With high resolution endoscope, continuous normal saline irrigation, and meticulously controlled hemostasis, there is almost no bleeding in the surgical filed. In both of our cases, there were severe adhesion between the cysts and the surrounding dura and nerve roots, possibly due to the inflammatory reaction induced by the cyst. The adhesion increased the risks for incidental dural tear or nerve root injury. However, with the UBE technique, we could perform delicate dissection to release the adhesion and gently manipulate around the neural structures. These unique features made the surgical procedure possible for en bloc excision of the cyst in a crystal clear and magnified surgical field with less complications.

For HDC, recurrence is a potential late complication. Although the origin of the cyst was believed to be the intervertebral disc, the concomitant discectomy was not recommended to prevent the recurrences [13]. HDC usually occurred in relatively young active people and discectomy might have negative impact on spinal biomechanics as well as to accelerate the degeneration process. Thermal annuloplasty using the radiofrequency wand might be an alternative technique to reduce the risks of recurrence and avoid the discectomy [19].

In our second case, we noted a residual cyst remnant in the follow-up MRI. The remnant located in the blind spot deep underneath the dura and was difficult to reach due to the broad base structure of the cyst. Fortunately, the remnant was asymptomatic; therefore, the secondary surgery was not necessary.

\section{CONCLUSION}

In conclusions, biportal endoscopic surgery is a safe, effective minimally invasive surgical treatment for HDC. En bloc excision of the cyst along with thermal annuloplasty could be considered as a choice for the surgical treatment.

\section{CONFLICT OF INTEREST}

No potential conflict of interest relevant to this article.

\section{REFERENCES}

1. Kobayashi S, Takeno K, Uchida K, Yayama T, Nakajima H, Miyazaki T, et al. Pathogenesis of the discal cysts communicating with an adjacent herniated disc. Histological and ultrastructual studies of two cases. Joint Bone Spine 2010; 77:184-186.

2. Sanjeevan R, Prabu S, Azizul A, Abdul-Halim Y. Discal cyst of the lumbar spine: case report of a rare clinical entity. Malays Orthop J 2018;12:56-58.

3. Bansil R, Hirano Y, Sakuma H, Watanabe K. Transition of a herniated lumbar disc to lumbar discal cyst: a case report. Surg Neurol Int 2016;7:S701-S704.

4. Hsu KY, Zucherman JF, Shea WJ, Jeffrey RA. Lumbar intraspinal synovial and ganglion cysts (facet cysts). Ten-year experience in evaluation and treatment. Spine (Phila Pa 1976) 1995;20:80-89.

5. Artico M, Cervoni L, Carloia S, Stevanato G, Mastantuono 
M, Nucci F. Synovial cysts: clinical and neuroradiological aspects. Acta Neurochir (Wien) 1997;139:176-181.

6. Hwa Eum J, Hwa Heo D, Son SK, Park CK. Percutaneous biportal endoscopic decompression for lumbar spinal stenosis: a technical note and preliminary clinical results. J Neurosurg Spine 2016;24:602-607.

7. Kim JE, Choi DJ, Park EJJ, Lee HJ, Hwang JH, Kim MC, et al. Biportal endoscopic spinal surgery for lumbar spinal stenosis. Asian Spine J 2019;13:334-342.

8. Pao JL, Lin SM, Chen WC, Chang CH. Unilateral biportal endoscopic decompression for degenerative lumbar canal stenosis. J Spine Surg 2020;6:438-446.

9. Kono K, Nakamura H, Inoue Y, Okamura T, Shakudo M, Yamada R. Intraspinal extradural cysts communicating with adjacent herniated disks: imaging characteristics and possible pathogenesis. AJNR Am J Neuroradiol 1999;20:1373- 1377.

10. Lee HK, Lee DH, Choi CG, Kim SJ, Suh DC, Kahng SK, et al. Discal cyst of the lumbar spine: MR imaging features. Clin Imaging 2006;30:326-330.

11. Kim SH, Ahn SS, Choi GH, Kim DH. Discal cyst of the lumbar spine: a case report. Korean J Spine 2012;9:114-117.

12. Jha SC, Higashino K, Sakai T, Takata Y, Abe M, Nagamachi A, et al. Percutaneous endoscopic discectomy via transforam- inal route for discal cyst. Case Rep Orthop 2015;2015:273151.

13. Park JW, Lee BJ, Jeon SR, Rhim SC, Park JH, Roh SW. Surgical treatment of lumbar spinal discal cyst: is it enough to remove the cyst only without following discectomy? Neurol Med Chir (Tokyo) 2019;59:204-212.

14. Aydin S, Abuzayed B, Yildirim H, Bozkus H, Vural M. Discal cysts of the lumbar spine: report of five cases and review of the literature. Eur Spine J 2010;19:1621-1626.

15. Yu HJ, Park CJ, Yim KH. Successful treatment of a symptomatic discal cyst by percutaneous C-arm guided aspiration. Korean J Pain 2016;29:129-135.

16. Kim JS, Choi G, Lee CD, Lee SH. Removal of discal cyst using percutaneous working channel endoscope via transforaminal route. Eur Spine J 2009;18 Suppl 2:201-205.

17. Kang H, Liu WC, Lee SH, Paeng SS. Midterm results of percutaneous CT-guided aspiration of symptomatic lumbar discal cysts. AJR Am J Roentgenol 2008;190:W310-W314.

18. Chen S, Suo S, Li C, Wang Y, Li J, Zhang F, et al. Clinical application of percutaneous transforaminal endoscopic surgery in lumbar discal cyst. World Neurosurg 2020;138:e665-e673.

19. Sairyo K, Kitagawa Y, Dezawa A. Percutaneous endoscopic discectomy and thermal annuloplasty for professional athletes. Asian J Endosc Surg 2013;6:292-297. 